\title{
RESPONSABILIDAD SOCIAL CORPORATIVA: Qué se hace y qué debe hacerse
}

\author{
David Solano \\ UNIVERSIDAD ESAN (PERÚ) \\ dsolano@esan.edu.pe
}

\section{Resumen}

La responsabilidad social empresarial o corporativa es uno de los principales temas de interés de los empresarios en el mundo, porque los mercados de consumo y de capitales premian o castigan la relación de las empresas e instituciones con su medio. Sin embargo, en la actualidad este abordaje es aún incipiente y presenta varias incorrecciones: es reactivo, excesivamente mediático, poco especializado y confunde filantropía o relaciones públicas con responsabilidad social. Refleja poco conocimiento no sólo de los conceptos básicos, sino también de la razón de ser de todo el proceso de responsabilidad social: la mejora constante de las relaciones con la población como base para el desarrollo sostenible. Para lograr esta meta es necesario trazar objetivos claros, definir adecuadamente el público objetivo y diseñar planes de acción estructurados y coherentes. Sólo así podremos evaluar el éxito o fracaso de nuestra intervención.

Palabras clave: responsabilidad social, márketing social, planificación.

\begin{abstract}
Corporate Social Responsibility has caught the attention of the business community worldwide because consumer and capital markets now champion or punish a company for its relationship with its environment. But this approach is still new and has several defects: it's reactive, excessively focused on mass media, it's not specialized, it confuses Public Relations with Social Responsibility, etc. This shows not only little knowledge of the basic concepts but also of the main reason underlying any Social Responsibility process: a sustainable improvement in the relationship with the population as a foundation for sustainable development. In order to achieve this it is necessary to have clear objectives, an clear definition of the target audience, and well designed action plans. Only then will we be able to assess the success or failure of our intervention.
\end{abstract}

Key words: social responsibility, social marketing, planning.

\section{Introducción}

La Responsabilidad Social Corporativa ${ }^{1}$ (RSC) se ha convertido en uno de los principales temas de atención de los empre- sarios en el mundo entero. Está demostrado que la sostenibilidad de los negocios está directamente relacionada con un buen manejo del entorno, lo reafirma el hecho de que los mercados de capitales

1 En este artículo se usará «Responsabilidad Social Corporativa» como sinónimo de «Responsabilidad Social Empresarial». 
en el mundo premian o castigan, según corresponda, el manejo del medio en el que se desarrolla la empresa.

Sin embargo, en el concepto sólo se explicita lo «social», a pesar de que el concepto de responsabilidad social incluye lo ambiental. Esta omisión se ha traducido en que muchas empresas dejen de lado el trabajo estratégico en los temas ambientales y deleguen su manejo a los técnicos. Por esta razón nos hemos atrevido a incluir la denominación «ambiental» en el concepto de RSC y, por lo tanto, a renombrarlo como «Responsabilidad Social y Ambiental Corporativa» (RSAC), término que será utilizado a lo largo de estas páginas.

El presente artículo parte de la hipótesis de que la mayoría de los procesos de RSAC en el Perú y en América Latina son eminentemente reactivos, no cuentan con una planificación ni un entendimiento pleno y aplicable de los conceptos que la motivan. En muchos casos se han convertido en herramientas de control y transacción social. Así planteados, no cumplen con su doble beneficio - a la empresa y a la comunidad, que hemos denominado público objetivo-, desvirtúan su principal objetivo: la convivencia armónica y la contribución al desarrollo sostenible, y terminan convertidos en meros ejercicios de filantropía o de transacción.

El artículo se inicia con la descripción, discusión y aclaración de los distintos conceptos de RSAC, los que luego se contrastan con la forma como son entendidos en su uso actual. Luego, y en función de dichos conceptos, se muestran los errores más frecuentes que se cometen en el desarrollo de procesos de RSAC.
A renglón seguido, analizamos la RSAC desde un enfoque estratégico orientado a resultados, con beneficios claros para la empresa y la sociedad. Para ello utilizamos conceptos de planificación estratégica y márketing social.

Este artículo forma parte de un libro (en proceso de edición) en el que se desarrollan planteamientos operativos para llevar a cabo la RSAC en una empresa o institución. La idea central es mostrar que la RSAC implica colaborar, partiendo de un esfuerzo individual, para lograr el desarrollo común. Esta tarea no es fácil y es muchas veces incomprendida, no sólo por los beneficiarios sino también por los funcionarios promotores. Para lograr la participación de todos los actores involucrados, ya sea dentro o fuera de la empresa, la RSAC debe ser completamente explícita en acciones y objetivos. Es decir, sólo con objetivos y tareas claras lograremos el compromiso empresarial y, por ende, una mayor probabilidad de generar relaciones positivas entre empresa y sociedad, base del desarrollo sostenible.

\section{Conceptos de RSAC}

\subsection{Qué es la RSAC}

Según el «Libro verde» difundido en la Comunidad Europea, la RSAC es la integración voluntaria de las preocupaciones sociales y medioambientales de las empresas e instituciones, en sus operaciones comerciales y en la relación con sus interlocutores.

La pregunta que surge aquí es: ¿quiénes son los interlocutores? La publicación de la Unión Europea (2001) le da respuesta cuando menciona que la RSAC tiene dos 
dimensiones: la interna y la externa. La dimensión interna tiene en cuenta los recursos humanos de la organización, sus objetivos personales, sus problemas y necesidades. Por supuesto, también a los accionistas, pero eso se da por descontado. Por otro lado, la dimensión externa incluye a las comunidades locales, los proveedores y los clientes.
- Ofrece productos y servicios que responden a las necesidades de sus usuarios, de tal manera que contribuyan a su bienestar.

- Tiene un comportamiento que va más allá del cumplimiento de los estándares mínimos reglamentarios.

La primera responsabilidad social y ambiental de la empresa es con sus trabajadores. No sólo debe cumplir con los beneficios que les corresponden, sino además estar expectante de sus necesidades personales, familiares y profesionales. Ninguna empresa o institución puede decir que realiza RSAC si no respeta primero a sus trabajadores, si no les proporciona ambientes de trabajo saludables y les da condiciones favorables para su desarrollo personal y profesional.

Según la definición del «Libro verde», dos elementos permiten determinar si una empresa o institución desarrolla acciones de RSAC. Primero, debe ser un acto voluntario; es decir, nadie podrá obligar a una empresa o institución a ser social y ambientalmente responsable. Segundo, la RSAC debe exceder el cumplimiento de la ley, estándar mínimo obligatorio que todos deben cumplir, tanto dentro como fuera de la empresa o institución. Esto desmiente la tendencia en América Latina de calificar como social y ambientalmente responsables a aquellas empresas que cumplen la ley, como si fuera un favor a la sociedad. Es más, son las mismas empresas quienes se califican como «muy buenas» porque cumplen con la ley.

La RSAC empieza cuando la empresa cumple no sólo lo legal, sino que excede dicho marco. Es decir, otorga más beneficios a la sociedad y a los trabajadores que aquellos a los que la obliga el marco legal existente.

Bestratén y Pujol (2004) mencionan que una empresa es social y ambientalmente responsable si:
- Demuestra conducta ética en todas sus acciones.

- Brinda condiciones saludables y seguras a sus trabajadores.

- Muestra respeto estricto por el ambiente, interno y externo.

- Está integrada a la comunidad, conoce y participa de sus anhelos y necesidades, así como de sus problemas.

Según Acción RSE (2001), para que una empresa sea considerada responsable socialmente debe desarrollar acciones en cinco ámbitos:

- Ética empresarial.

- Medio ambiente.

- Compromiso con la comunidad.

- Márketing responsable.

- Calidad de vida laboral.

\subsection{Importancia de la RSAC para la empresa o institución}

Lo primero que debe tener en cuenta una empresa o institución es que la RSAC no 
La diferencia entre relaciones públicas, filantropía y RSAC es evidente. La filantropía es el gasto en asuntos particulares, sin esperar retorno alguno. Las relaciones públicas consisten en una relación reactiva hacia la comunidad; la empresa se da a conocer y ayuda a la solución de las demandas de la población. Si bien implica ya un compromiso, este sólo es de carácter puntual y básicamente relacionado con los temas de interés para la empresa. La RSAC es una relación proactiva ante las necesidades de desarrollo de la sociedad. La empresa se anticipa a conocer esas necesidades para atenderlas mediante un compromiso sostenible; es decir, de largo plazo.

es un gasto, sino una inversión. La RSAC proporciona los siguientes beneficios:

- Disminuye los costos por paralización de operaciones o seguridad, al reducir la posibilidad de conflictos con la población.

- Mejora la productividad, al contar con trabajadores motivados o con mejores condiciones de salud, lo cual contribuye a incrementar su fidelidad con la empresa.

- Mejora el ambiente interno y la seguridad de los trabajadores, al considerar sus necesidades.

- Mejora el ambiente externo, al identificar y mitigar los impactos que sus actividades pueden generar en el entorno.

- Brinda una imagen de responsabilidad en la empresa.

\subsection{Qué es y qué no es la RSAC}

Por lo general, la RSAC se confunde con la filantropía o con la caridad. Así, se la utiliza para «lavar la cara» de la empresa respecto de errores que haya cometido en el pasado, ya sea de carácter social o ambiental; por ejemplo, contaminación o resquebrajamiento social causados por una operación poco cuidadosa. Asimismo, las acciones dirigidas a la sociedad se conciben con fines de relaciones públicas. Se «vende» la imagen de una empresa sensible a las necesidades de la población.

Bestratén y Pujol (2004) relacionan compromiso social e interés consciente de la empresa para definir las diferentes formas de intervención social y ambiental de las empresas. A continuación, presentamos dicha relación:

\begin{tabular}{|c|lll|}
\hline \multirow{2}{*}{$\begin{array}{r}\text { ALTO } \\
\text { Compromiso social } \\
\text { sostenible }\end{array}$} & Filantropía & $\begin{array}{l}\text { Economía social } \\
\text { convencional }\end{array}$ & RSAC \\
\cline { 2 - 4 } BAJO & Caridad & $\begin{array}{l}\text { Relaciones } \\
\text { Públicas }\end{array}$ & $\begin{array}{l}\text { Márketing } \\
\text { con causa }\end{array}$ \\
\cline { 2 - 4 } & $\begin{array}{l}\text { Fracaso } \\
\text { seguro }\end{array}$ & Negocio puro & $\begin{array}{l}\text { Manipulación } \\
\text { social }\end{array}$ \\
\hline & Bajo & $\begin{array}{l}\text { Interés } \\
\text { consciente de } \\
\text { la empresa }\end{array}$ & Alto \\
\hline & & & \\
\hline
\end{tabular}

Adaptada de Bestartén y Pujol (2004: 4). 
Es importante resaltar que la RSAC necesita un alto compromiso social sostenible y un alto interés consciente de la empresa. En el otro extremo, en el cuadrante inferior derecho, el alto interés consciente y el bajo compromiso dan como resultado una estrategia de manipulación social de la empresa para el logro de sus propios fines, actitud reñida con la ética. Las relaciones públicas se ubican en el cuadrante de medio compromiso social y medio interés consciente.

Por su parte, el márketing con causa consiste en la publicidad que realiza la empresa con el fin de promover alguna causa social o ambiental. Ejemplo de ello son las campañas para promover el ahorro del agua. Con mucha frecuencia, el márketing con causa también se confunde con la RSAC y constituye una de las formas más usadas por las empresas para asumir el tema.

La decisión consciente de la empresa de realizar filantropía o márketing con causa no le genera problema alguno. El verdadero problema surge cuando la empresa invierte en cualquiera de las dos opciones y espera resultados equivalentes a los que habría obtenido con la aplicación de una estrategia de RSAC.

\subsection{La ética como factor fundamental de la RSAC}

Un elevado compromiso con la comunidad implica una alta dosis de ética en la acción empresarial. No existe RSAC sin ética, aun si se realizan inversiones millonarias.

¿Qué es la ética? Giraldo (2001:1) la define como «el desarrollo propio, sin atentar contra el libre desenvolvimiento de los demás, es decir es meramente subjetivo». Esto significa desarrollarse, pero sin perjudicar en ese desarrollo a otras personas o instituciones.

Por lo tanto, un comportamiento ético consiste no sólo en evitar y combatir la corrupción, como se concibe normalmente, sino en respetar las libertades, los anhelos y las potencialidades de los demás. Según López (2001), ello implica que si en la práctica una empresa quiere actuar en forma ética debe:

- Vivir de acuerdo con cinco valores fundamentales: igualdad, libertad, diálogo, respeto y solidaridad.

- Tener valores comunes para sus miembros.

- Luchar por alcanzar la satisfacción de todos los agentes involucrados, internos y externos, directa o indirectamente.

- Asumir la responsabilidad de sus actuaciones.

- Respetar la palabra antes que lo escrito.

Si bien estos cinco principios pueden ser adaptados para el individuo, si pensamos en los directivos y trabajadores de una institución añadiríamos otro principio: «Ser coherente y predicar con el ejemplo».

Sin embargo, la ética no sólo debe estar presente en las relaciones externas (por ejemplo, combatir la corrupción) de la empresa, sino también en el trabajo que se desempeña al interior de ella. En consideración a esto, y no siendo este un artículo sobre ética, asumiremos implícitamente que cada paso que conduce a una estrategia y una acción de RSAC es eminentemente ético. De no ser así, ya no estaríamos ante una estrategia de RSAC. 


\subsection{Tipos de RSAC}

En el tema de la RSAC, como en muchos otros, es frecuente preguntarse por dónde comenzar. Bestratén y Pujol (2004) presentan una clasificación de responsabilidad social que incluye tres categorías: primarias, secundarias y terciarias, y nos permite tener una respuesta. Estas responsabilidades son un continuo, es decir, no se puede (o no se debe) pasar a las responsabilidades secundarias sin haber abordado las primarias.

Las responsabilidades primarias son inherentes a la misma actividad, razón por la cual no pueden considerarse aún acciones propias de RSAC. Sin embargo, constituyen la mejor forma de empezar a desarrollarlas. Entre las principales podemos citar:

- Brindar productos de calidad a precios justos.

- Crear riqueza en la empresa y en la sociedad.
- Respetar los derechos de los trabajadores y darles condiciones de trabajo dignas, que favorezcan su desarrollo personal y profesional.

- Promover una empresa sostenible y en crecimiento.

- Identificar y mitigar o evitar los impactos negativos sobre el ambiente.

- Cumplir las leyes y reglamentos que le sean aplicables.

- Distribuir equitativamente la riqueza generada.

Las responsabilidades secundarias son aquellas que exceden lo obligatorio y cuya aplicación requiere esfuerzos de la empresa. Podemos decir que este es el umbral entre la RSAC y la obligatoriedad. Bestratén y Pujol (2004) dividen estas responsabilidades entre aquellas que se contraen con los trabajadores y aquellas que configuran las relaciones con la comunidad.

\section{Responsabilidades secundarias}

Con los trabajadores

Con la comunidad

- Ofrecer calidad de trabajo en equilibrio con la vida extralaboral.

- Favorecer la iniciativa, la autonomía y la creatividad en el trabajo.

- Proporcionar empleos estables, en lo posible.

- Favorecer el crecimiento intelectual y profesional de los trabajadores.

- Facilitar asistencia para la salud, más allá de lo exigible.

- Aportar información sobre la empresa en todos los aspectos, en lo posible.

- Reconocer el esfuerzo y los logros alcanzados.
- Dar empleo a la comunidad local.

- Mejorar el ambiente más allá de sus propias operaciones.

- Publicitar con sus productos valores para un entorno social más humano.

- Facilitar la integración de personas con habilidades especiales.

- Ayudar a los proveedores a mejorar sus servicios.

- Asesorar a la comunidad en materias en las cuales la empresa tenga conocimientos especializados.

- Colaborar con la formación de jóvenes profesionales. 
En cuanto a las responsabilidades terciarias, son aquellas que no están incluidas en la actividad específica de la empresa. En la práctica, muchas de ellas son desarrolladas por las empresas antes de asumir las responsabilidades primarias o secundarias, porque se relacionan con mayor facilidad con lo social y causan un mayor impacto en el observador externo, como los medios de comunicación. Entre ellas podemos citar:

- Proporcionar a la población ayuda para comidas fuera del hogar (ejemplo, comedores populares).

- Proporcionar a la población ayuda para vivienda (urbanizaciones, agua, alcantarillado, etc.).

- Facilitar a los pobladores medios para el disfrute de actividades culturales y recreativas (cines, parques, etc.).

- Promover programas de ayuda a la comunidad o a otros.

- Colaborar con centros de formación, como escuelas, universidades, entre otros.

- Ayudar a la construcción de infraestructura social.

- Mecenazgo en actividades culturales o deportivas.

Si bien estas actividades son necesarias, bajo ningún concepto deben signifi- car el abandono de las responsabilidades primarias o secundarias, como muchas veces ocurre. Desarrollarlas no justifica, de modo alguno, descuidar lo primario ni lo secundario.

\section{Errores y resultados de una concepción equivocada de RSAC}

Expuestos y analizados los conceptos sobre RSAC, toca ahora contrastarlos con la realidad. Creemos que las empresas e instituciones incurren, con frecuencia, en errores al desarrollar acciones de RSAC, los cuales suelen generar efectos negativos en la empresa y en la sociedad.

\subsection{Errores de concepción}

- Falta de claridad en determinar el interés concreto de la empresa en la $R S A C$. Se deben definir los objetivos de RSAC que la empresa se traza en función de sus necesidades, primarias y secundarias, y las de la sociedad. La RSAC requiere una decisión consciente sobre la base de objetivos claros, que deben estar en concordancia con los objetivos de la empresa en el corto, mediano y largo plazo. No interiorizar esta relación significa realizar acciones desvinculadas, que no tendrán efecto alguno en la acción institucional.

\footnotetext{
Teniendo en cuenta la definición que se está empleando, la política de RSAC de una entidad gubernamental debe estar más arraigada que la de una entidad privada, tanto en lo interno (con sus trabajadores y sus productos-servicios) como en lo externo (implicancias ambientales o impacto en la comunidad de sus decisiones y sus acciones, y generación de riqueza para la sociedad), así como con relación a las responsabilidades primarias, secundarias o terciarias. Este es un concepto muy poco interiorizado en las entidades públicas latinoamericanas.
} 
Por ejemplo, en la mayoría de reportes institucionales de responsabilidad social que elaboran las empresas, se puede apreciar la ausencia de planes estratégicos en el desarrollo de la RSAC. Los informes son sólo un listado de acciones evaluadas en función del monto invertido y del impacto mediático alcanzado; no se toma en cuenta el impacto en la solución de problemas concretos y la sostenibilidad de dichas acciones. Es más, muchas veces las acciones se presentan desvinculadas entre sí, dando la impresión de que la empresa no tiene un norte definido respecto de la RSAC.

- Confundir las Relaciones Públicas con RSAC. En este caso, utilizar la RSAC como una forma de promover la imagen de la empresa constituye el principal objetivo. Si bien, por definición, la empresa es libre de decidir si desarrolla RSAC, debe ser consciente de las diferencias entre esta y las relaciones públicas. Los problemas empiezan cuando la empresa se introduce en el mundo social sin objetivos claros y se convierte en «mecenas» ante las demandas dispersas de la población. Este es un error muy común y genera inversiones sin «retorno» alguno, en términos de los objetivos de RSAC que se ha planteado la empresa.

RSAC efectiva $=$ Logro de los objetivos de RSAC = Contribución al logro de objetivos empresariales

- Aplicar recurrentemente la «política de bombero». Es decir, apagar los incendios que genera el actuar con poco conocimiento de la realidad social. La empresa debe tener los objetivos de RSAC claramente definidos e interiorizados a fin de definir las acciones que se deben desarrollar a largo plazo, para lograr que la RSAC sea efectiva y ayude al desarrollo de las comunidades.

- Escasa capacidad e interés en la identificación de las necesidades y demandas de la población. Cometer este error convierte a la empresa en reactiva. Se debe tener presente, en todo momento, que la RSAC implica una relación sostenible y proactiva con la población (involucrarse constantemente) y de lectura anticipada de sus necesidades. Es decir, adelantarse y ayudar a la población (no imponer) a definir sus necesidades y objetivos. Sin un conocimiento de dichas necesidades y demandas, no se puede definir líneas de ayuda que impliquen un mayor impacto de las inversiones realizadas, ya sea en dinero, bienes o tiempo.

- No difundir internamente el concepto de compromiso. El compromiso es un elemento intrínseco a la RSAC e involucra no sólo al equipo promotor (por ejemplo, el equipo de Relaciones Comunitarias o Ambientales), sino también a la empresa en su totalidad. Es decir, empieza en la alta dirección y continúa por los diferentes niveles de la empresa. Sin compromiso no hay RSAC, postura que cede el lugar a la filantropía. Las acciones de RSAC le dan otra dimensión a la inversión, pues esta se convierte en parte de un proceso de colaboración consciente para el desarrollo de la sociedad.

- Descuidar la promoción y la interiorización de la RSAC en los miembros de la empresa. Si el concepto de compromiso está interiorizado, cada traba- 
jador debe convertirse en un ejemplo de ese compromiso con la sociedad. De lo contrario, la sociedad percibirá la relación de forma negativa y los esfuerzos de RSAC que desarrolla la empresa podrían fracasar. Por ejemplo, la falta de compromiso se manifiesta en temas tan nimios como la exigencia de los trabajadores de obtener un trato preferencial en el uso de servicios públicos o en la relación con la autoridad.

- Confundir cumplimiento de lo legal con la RSAC. El cumplimiento de las leyes es promocionado por las empresas como sinónimo de gran responsabilidad. Sin embargo, conceptualmente la RSAC significa exceder lo legal para brindar más de lo exigido para el desarrollo de la sociedad.

- Confundir filantropía con RSAC o buscar el control de la población. Este hecho implica que las acciones desarrolladas tengan un efecto contrario al deseado por la RSAC, ya que genera una desconfianza institucionalizada de la población hacia la empresa, así como un resquebrajamiento entre los beneficiados y quienes se sienten excluidos.

- Inadecuada conformación de los equipos. Se debe tratar de que las personas encargadas de la RSAC en una empresa o institución cuenten con la preparación adecuada, tengan la sensibilidad social necesaria, sean capaces de percibir las necesidades de la población, «hablen el mismo idioma», tengan carisma y sean percibidas como personas creíbles, de confianza. Mantener una persona sin las características necesarias, que cree que su función es negociar con la población para darle lo menos posible, genera un efec- to contrario al buscado. Por lo general, los temas de RSAC son difíciles de explicar. Por ello, la diferencia entre una RSAC efectiva y otra no efectiva es, muchas veces, la capacidad del equipo responsable de identificarse con la población.

- Definición poco clara de los objetivos de las acciones de RSAC o no asimilada por los trabajadores. Es decir, pregonar que se realizan acciones de RSAC, mientras que en dicho proceso se usan herramientas para transar con la población de tal manera de lograr que esta acepte ciertas decisiones. Esto significa que no se tiene claro qué se quiere lograr y, además, ignorancia respecto de la RSAC. Esta forma de abordar la RSAC es ineficiente e ineficaz, pues cuando estos «acuerdos» se producen surgen más exigencias, reales o fingidas, porque la población percibe que la empresa la necesita y está dispuesta a dar lo que sea por lograr su respaldo.

- Escasa claridad en la definición de contaminación ambiental. El concepto de ambiente que se utiliza en la actualidad va más allá de lo referido a los recursos naturales (creer que si no se contamina el agua, el aire u otro elemento, no existe un impacto en el ambiente), incluye también las relaciones de las personas con los recursos (cómo se organizan para aprovecharlos) y las relaciones entre las personas. Por ello, por ejemplo, aunque no se genere contaminación de los recursos, si se polariza a la población (lo que están a favor y en contra) y esta percibe a la empresa como contaminadora, ya se generó un problema ambiental que debe ser enfrentado. 
Hay una máxima muy poco aceptada y respetada en la gestión ambiental empresarial, pero muy cierta: «Si la empresa no contamina, pero la gente cree que contamina, la empresa tiene un problema ambiental que debe enfrentar y solucionar».

\subsection{Consecuencias de los errores de concepción}

A continuación, se presentan algunos «síntomas» que deben tenerse en cuenta y cuya aparición debe llevar a pensar si la política de RSAC que lleva a cabo la empresa está adecuadamente definida y operativizada o se está cometiendo los errores mencionados en el punto anterior.

- Mala relación con la población. Se manifiesta en situaciones de tensión permanente, con acusaciones mutuas de responsabilidad frente a diversos hechos, sea por acción u omisión.

- Imagen de la empresa como donador frecuente, sin efectos visibles en la relación con la comunidad. Los pobladores consideran que la empresa tiene la obligación de financiar sus necesidades, pues perciben que es la culpable de todos los males, ya sea por conveniencia o «comodidad». Esta relación no es permanente ni de confianza, más bien es una relación de clientelismo que la empresa prefiere mantener para evitar el riesgo de exacerbar una situación de tensión permanente.

- Percepción de la población de que la empresa es responsable de todos los males de la ciudad. Esta percepción se manifiesta tanto pública como privadamente por la comunidad, sin temor alguno de caer en inexactitudes.
Frente a ello, las empresas adoptan dos posturas. La primera consiste en «dejar pasar» estas acusaciones, lo cual incrementa las críticas, mientras que la segunda consiste en responder con demandas legales, que también deterioran la relación y convierten a la empresa (en la percepción popular) en enemigo público de la libertad de expresión.

- Incertidumbre permanente respecto de la reacción de la población frente a la adopción o no de una determinada acción. Esto sucede cuando no existe una actitud proactiva para conocer y anticiparse a los problemas y necesidades de la población.

\section{3. ¿Qué debe lograr la empresa con la RSAC? Las campañas de cambio social}

El hecho de confundir filantropía con RSAC puede llevar a pensar que la labor de las empresas o instituciones es financiar obras o ser promotoras de obras. Para cambiar esta percepción, las empresas tienden a responder que «no son el Estado», que cumplen con pagar sus impuestos y sus obligaciones para que el Estado invierta en las obras necesarias para la población.

La RSAC implica no sólo que la empresa ayude a generar las condiciones para el desarrollo sostenible, tanto de la propia empresa como de la población, sino también que promueva el cambio social. Sin embargo, esto no significa que la empresa realice el cambio. Este objetivo les corresponde a los pobladores, quienes con la ayuda de la empresa, mediante campañas de cambio social, encontrarán el camino para lograrlo. 
Kotler y Roberto (1992: 7) definen campaña de cambio social como «el esfuerzo organizado, dirigido por un grupo (el agente de cambio), que intenta persuadir a otros (adoptantes objetivo) para que acepten, modifiquen o abandonen ciertas ideas, actitudes, prácticas y conductas». Desde el punto de vista de la RSAC, la empresa debe convertirse en el agente de cambio o en el promotor del funcionamiento de un grupo agente de cambio.

Este concepto es radicalmente opuesto a aquello que las empresas latinoamericanas, en la mayoría de los casos, desarrolla como RSAC. Podemos concluir que toda estrategia de RSAC debe ser planteada como una estrategia de cambio social que incluya lo siguiente:

- Objetivos claros respecto de lo que se quiere solucionar o aprovechar. Además, estos objetivos deben ser consensuados y compartidos.

- Un agente de cambio claro y reconocido.

- Adoptantes objetivo claramente identificados.

- Canales. Las formas institucionales, mediáticas o comunicacionales que tomarán las acciones que desarrollarán los adoptantes objetivo.

- Estrategia de cambio basada, fundamentalmente, en una estrategia de márketing social.

- Estrategia de sostenibilidad. Es decir, que perdure en el tiempo.

De acuerdo con este concepto y también según Kotler y Roberto (1992), para que una campaña de cambio social tenga éxito en su fase de aplicación, debe cumplir con las siguientes características:
- Fuerza. Es decir, una motivación anterior al mensaje. Las oportunidades o los problemas deben ser considerados importantes antes de iniciarse la campaña. Si este interés no existe, debe trabajarse primero en crearlo.

- Dirección. La población debe tener muy claro cómo contribuirá al cambio.

- Mecanismo. La institución o el grupo que lidera el cambio debe ser fácilmente identificable.

- Eficiencia y eficacia. La institución o el grupo que lidera el cambio debe ser, en la realidad y en la percepción popular, eficaz y eficiente antes, durante y después de la campaña.

- Costo-beneficio. La población debe ser consciente de que frente a lo que va a recibir, bien vale la pena un sacrificio.

La RSAC busca promover el cambio para lograr el desarrollo sostenible. Esto es diferente a realizar el cambio o a financiar el cambio, como se asume comúnmente en las empresas. Ningún agente, público o privado, podrá generarlo por sí solo. Lograr una RSAC eficaz y eficiente exige de la empresa un replanteamiento de sus objetivos, así como el cultivo de sus relaciones internas y externas.

\section{Cómo generar un proceso de RSAC que ayude a lograr el objetivo final: el desarrollo sostenible de la empresa $y$ de la sociedad}

Por lo general, el concepto de desarrollo sostenible ha sido poco entendido y asimilado por el sector empresarial. Dado que este concepto fue muy utilizado por los ambientalistas, se ha impregnado de los anticuerpos de ciertos grupos que con- 
sideran que trabajar el tema ambiental es un lujo que los países en desarrollo, que necesitan producir y generar empleo, no pueden darse.

En la segunda mitad de los años noventa, la Comisión Bruntland (así denominada por el apellido de la presidenta de dicho grupo de trabajo), nombrada por la ONU, definió el desarrollo sostenible como aquel que se genera hoy, pero sin poner en riesgo los recursos y el desarrollo de las generaciones futuras.

Otra definición señala que el desarrollo sostenible es aquel que toma en cuenta el desarrollo económico, el desarrollo social y el desarrollo ambiental. Esta definición es la que nos parece más práctica y operativa, pues indica qué debe hacer una empresa, un país, un gobierno local, entre otros, para lograr un desarrollo que perdure en el tiempo.

Nadie puede lograr el desarrollo sostenible si no cuenta y genera los recursos económicos adecuados para invertir (desarrollo económico), si sus propuestas no tienen el respaldo social e institucional (desarrollo social-institucional) y si no cuida sus recursos naturales y la calidad de su ambiente (desarrollo ambiental). Por ello, la gestión social y ambiental no es un favor que se hace a la sociedad, sino, más bien, una necesidad de todo aquel que desea lograr resultados que perduren.

El objetivo de todo agente económico y de todo Gobierno (nacional o subnacional) debe ser lograr el desarrollo sostenible. Esto refuerza la necesidad de que la empresa cuente con estrategias adecuadas y coherentes de RSAC.
Sobre esta base, el objetivo de la RSAC es generar desarrollo sostenible para la empresa y para la sociedad. En esa dirección, toda propuesta de RSAC debe contener en forma muy clara lo siguiente:

- Objetivos y metas por lograr.

- Público objetivo.

- Definición

- Necesidades

- Características

- Comportamientos esperados

- Estrategia de aplicación que, basada en el márketing social, pueda responder a las siguientes preguntas:

- ¿Con qué propuesta técnica lograremos el objetivo? (Producto)

- ¿Quién lo implementará? (Canales)

- ¿Qué resistencias puede haber del público objetivo? (Precio)

- ¿Cómo convencer al público objetivo? (Comunicación)

Es fundamental que todo proceso de RSAC tenga definidos estos aspectos antes de iniciarse. Sin embargo, y tal como lo mencionamos en páginas anteriores, es muy común no sólo no contar con una estrategia de aplicación, sino inclusive no tener objetivos. Y como en todo proceso, si no se cuenta con objetivos claros y estrategias de implementación, el fracaso será total, no sólo porque no se sabrá qué se quiere lograr (base para evaluar el éxito o fracaso de la intervención) sino, además, cómo y hacia dónde se deben canalizar los esfuerzos institucionales con el fin de tener un proceso de RSAC que logre una mejor relación con la población y que contribuya al desarrollo sostenible de la empresa y la sociedad. 


\section{Conclusiones}

Con el correr de los años, las empresas privadas han demostrado estar a la vanguardia en temas de gerencia. Sin embargo, en la gestión de la RSAC, en la mayoría de los casos, su accionar ha sido improvisado y poco profesional. Por ello, es necesario replantear la acción de la RSAC, otorgándole el carácter estratégico que necesita. Es muy importante que la empresa asuma un compromiso consciente, que comienza por dar a sus trabajadores las mejores condiciones de trabajo.

A través de un proceso que busque el cambio, la RSAC debe ser la herramienta mediante la cual la empresa genere las condiciones necesarias para su desarrollo y el de la población.

\section{Referencias bibliográficas}

ACCIÓN RSE. 2001. Indicadores de responsabilidad social corporativa. Santiago de Chile: Acción RSE, oct.

BESTRATÉN, Manuel y PUJOL, Luis. 2004. Responsabilidad social de las empresas (I y II). Madrid: Ministerio de Trabajo y Asuntos Sociales de España, Centro Nacional de Condiciones de Trabajo [www.mtas.es/inst./ntp/ntp_643.htm].

GIRALDO, María Elena. 2001. La ética en la administración [www.gestipolis.com].

KOTLER, Philip y ROBERTO, Eduardo. 1992. Márketing social: estrategias para cambiar la conducta pública. Madrid: Díaz de Santos. 467 págs.

LÓPEZ, Carlos. 2001. La ética como fuente de ventajas. IESE-Universidad de Navarra [www.gestipolis.com].

OEPEN, Manfred y HAMACHER, Winfred (eds). 2000. Communicating the environment: Environmental communication for sustainable development. Environmental Education, Communication and Sustainability-GTZ. Frankfurt: Peter Lang Inc. 294 págs.

PERÚ. CONSEJO NACIONAL DEL AMBIENTE. (CONAM). 1999. Manual de capacitación en manejo y resolución de conflictos ambientales. Lima: Conam.

SABATINI, Francisco. 1995. ¿Qué hacer frente a los conflictos ambientales? Ambiente y Desarrollo. Santiago de Chile, mar., vol. $\mathrm{XI}, \mathrm{n}^{\circ} 1$.

SCHWALB, María Matilde y MALCA, Óscar. 2004. Responsabilidad social: fundamentos para la competitividad empresarial y el desarrollo sostenible. Lima: Yanacocha, ALAC, CIUP. 249 págs.

SOLANO, David. 2005. Educación ambiental y gestión ambiental: algunos aspectos teóricos y metodológicos. Material del Curso Gestión Ambiental para el Desarrollo Sostenible Local. Lima: ESAN.

——. 2001. Comunicación y generación de conciencia ambiental. Tópicos en Educación Ambiental. México D. F., abr., vol. 3 , n. ${ }^{\circ}$, págs. 52-57.

UNIÓN EUROPEA. 2001. Libro verde: fomentar un marco europeo para la responsabilidad social de las empresas. Bruselas: Comisión de las Comunidades Europeas [www.europa.eu.int/comm/off/ green/index-es.htm]. 